\title{
Robot-assisted laparoscopic rectovaginopexy for rectal prolapse: a prospective cohort study on feasibility and safety
}

\author{
Werner A. Draaisma · Dorothée H. Nieuwenhuis · \\ Lucas W. M. Janssen · Ivo A. M. J. Broeders
}

Received: 14 September 2007 / Accepted: 5 December 2007 / Published online: 4 January 2008

(C) The Author(s) 2007

\begin{abstract}
Robotic systems may be particularly supportive for procedures requiring careful pelvic dissection and suturing in the Douglas pouch, as in surgery for rectal prolapse. Studies reporting robot-assisted laparoscopic rectovaginopexy for rectal prolapse, however, are scarce. This prospective cohort study evaluated the outcome of this technique up to one year after surgery. From January 2005 to June 2006, 15 consecutive patients with a rectal prolapse, either with or without a concomitant rectocele or enterocele, underwent robot-assisted laparoscopic rectovaginopexy with support of the da Vinci robotic system. A prospective cohort study was performed on operating times, blood loss, intra-operative and post-operative complications, and outcome at a minimum of one year after surgery. Median age at time of operation was 62 years (33-72) and median body mass index 24.9 (20.9-33.9). Median robot set-up time was $10 \mathrm{~min}(3-15)$ and median skin-to-skin operating time was $160 \mathrm{~min}(120-180)$. No conversions to open surgery were necessary. No in-hospital complications occurred and there was no mortality. Median hospital stay was four days (2-9). During one year follow-up, two patients needed surgical reintervention. One patient was operated for recurrent enterocele and rectocele one week after surgery. In another patient an incisional hernia at the
\end{abstract}

W. A. Draaisma - D. H. Nieuwenhuis · L. W. M. Janssen

I. A. M. J. Broeders $(\square)$

Department of Surgery,

University Medical Centre Utrecht,

Heidelberglaan 100, H.P. G04.228,

P. O. Box 85500, 3508, GA Utrecht, The Netherlands

e-mail: i.broeders@umcutrecht.nl

W. A. Draaisma

e-mail: wernerdraaisma@yahoo.com camera port occurred three months after surgery. At one year after surgery, $87 \%$ of patients claimed to be satisfied with their postoperative result. Robot-assisted laparoscopic rectovaginopexy proved to be an effective technique with favourable outcomes in most patients in this prospective series. The operating team experienced the support of the robotic system as beneficial, especially during the dissection of the rectovaginal plane and suturing in the Douglas pouch.

Keywords Rectal prolapse - Rectopexy ·

Rectovaginopexy $\cdot$ Laparoscopy $\cdot$ Robotic surgery $\cdot$ Robot

\section{Introduction}

Rectal prolapse, or rectal procidentia, is a disabling condition that is often associated with faecal incontinence and constipation. It is defined as protrusion of (a part of) the rectum beyond the anus [1]. Concurrent pelvic floor descent and prolapse of other pelvic floor organs such as the uterus or the bladder can often be identified. Rectal prolapse is presented infrequently, but the true incidence is unknown due to under reporting, especially in the elderly population [2]. Peaks in occurrence are noted in the fourth and seventh decades of life, and most patients (80-90\%) are women [3].

Abdominal rectopexy has emerged as procedure of choice for full-thickness rectal prolapse and its role as a transabdominal solution for this disorder has emerged even further with the introduction of minimally invasive techniques [4-9]. Even though laparoscopic surgery has been established for over a decade, laparoscopic colorectal procedures have taken longer to evolve as the skills required are advanced and take more time to master. In recent years, several studies have shown the benefits of laparoscopic 
rectopexy in comparison to open procedures in terms of postoperative pain, procedure-related complications, hospital stay, and return to daily routine, at the expense of a longer operation [10-13].

Robotic surgery was introduced in 1998 to overcome technical difficulties in visualisation and manoeuvrability in endoscopic surgery, especially in complex surgical procedures requiring extensive dissection and suturing in a small defined space [14]. Application of robotic surgery to patients suffering from full-thickness rectal prolapse has been initiated recently and will be addressed in this study.

\section{Materials and methods}

Between January 2005 and June 2006, 15 patients underwent robot-assisted laparoscopic rectovaginopexy. Patients referred were evaluated by medical history, physical examination, and rectal inspection during squeezing, including a digital rectal investigation. Furthermore, additional examination was performed by video defaecography in all patients. Anal endosonography and anal manometry were performed to determine a possible sphincter defect and anorectal function, respectively.

Clinical data including preoperative work-up, intra-operative and post-operative course, and follow-up at a minimum of one year after surgery were collected prospectively.

\section{Surgical technique}

All procedures were performed with the da Vinci robotic system, consisting of a console and of a three-armed cart which is positioned at the operating table. The patient is positioned in a modified Lloyd-Davis position with the legs widely abducted and with minimal hip flexion to accommodate the robotic cart. A sponge is placed on a clamp in the vagina to identify the fornix posterior during surgery.

A minimum of four ports were used in all procedures (Fig. 1). A $12 \mathrm{~mm}$ port hosted the camera and two $8 \mathrm{~mm}$ ports facilitated the robotic instruments. The camera and robotic instrument ports were positioned in a triangular fashion, similar to the basic ergonomic concepts of standard endoscopic surgery. Ports for assistance and exposition were positioned in the area between the camera and the robotic arm ports, slightly more distal from the target. After placing the ports, the robot was positioned and connected (Fig. 2). The operating surgeon left the sterile field at this point of time in order to work from the console, which was positioned at 3-4 m from the sterile field. In the University Medical Centre Utrecht all minimally invasive parts of this surgical procedure are performed by one of two experienced laparoscopic surgeons in this field (IB, LJ).

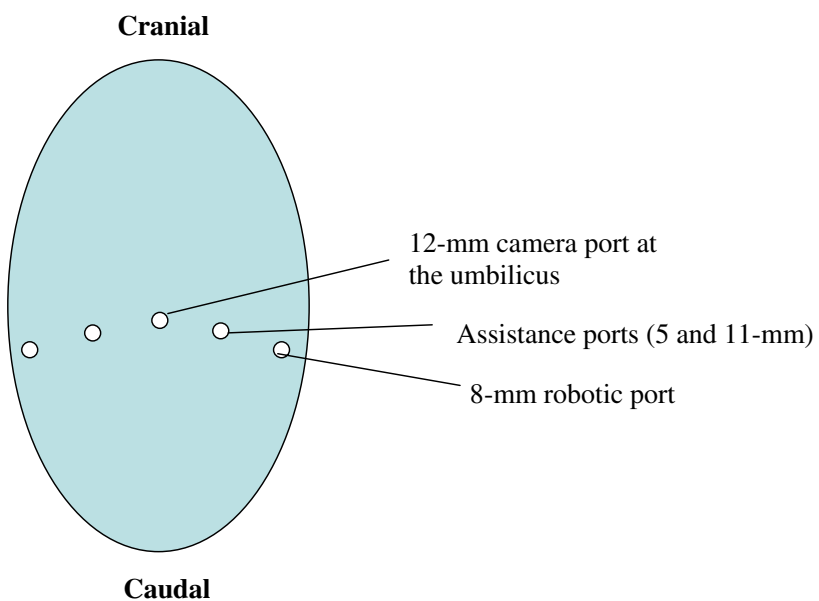

Fig. 1 Positioning of the robotic camera port, 8-mm robotic instrument ports, and standard laparoscopic assistance ports for robot-assisted rectovaginopexy

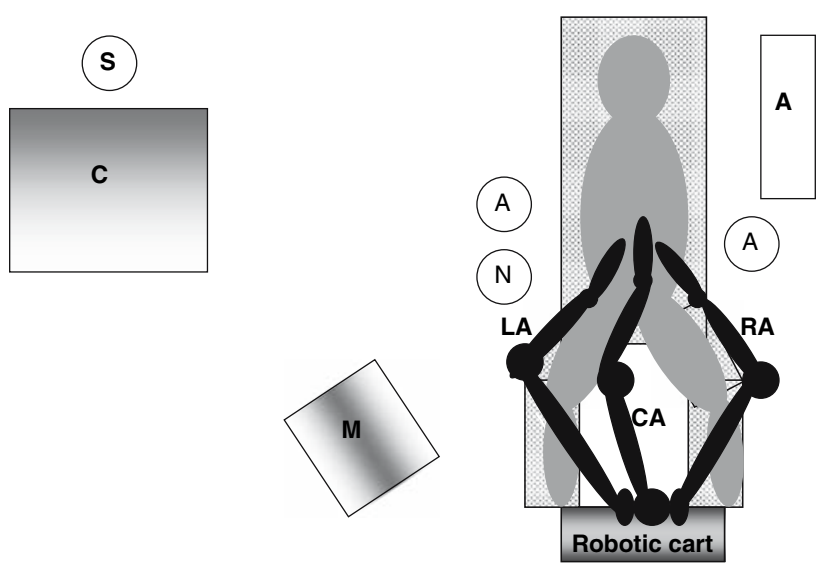

Fig. 2 Set up and positioning of the operating team in relation to the ports and robotic cart in robot-assisted laparoscopic rectovaginopexy for rectal prolapse. $A$, anaesthetic equipment, $R A$, robotic instrument arm, $C A$, camera arm, $M$, video cart (with monitor, insufflator, ultrasonic dissection generator, light source, camera unit, and focus control and synchronizer), $A$, table-side assistant, $N$, scrub nurse, $C$, robotic console, and $S$, surgeon

Furthermore, one experienced surgical assistant is present at the table-side.

All patients underwent a rectovaginopexy, a technique with the objective of avoiding damage to the hypogastric plexus and pelvic autonomic nerves by dissection of the peritoneum only, without mobilization of the dorsolateral side of the rectum and with preservation of the lateral ligaments (Fig. 3) [15].

Pneumoperitoneum was established using an open technique at the umbilicus and a blunt-tip disposable 12-mm port was introduced (Tyco Healthcare B.V., Zaltbommel, The Netherlands). The abdomen was insufflated with carbon dioxide to a pressure level of $12 \mathrm{~mm} \mathrm{Hg}$. A 30-degree camera was used and, after port placement and explorative laparoscopy, the patient was tilted to about $30^{\circ}$ head down 


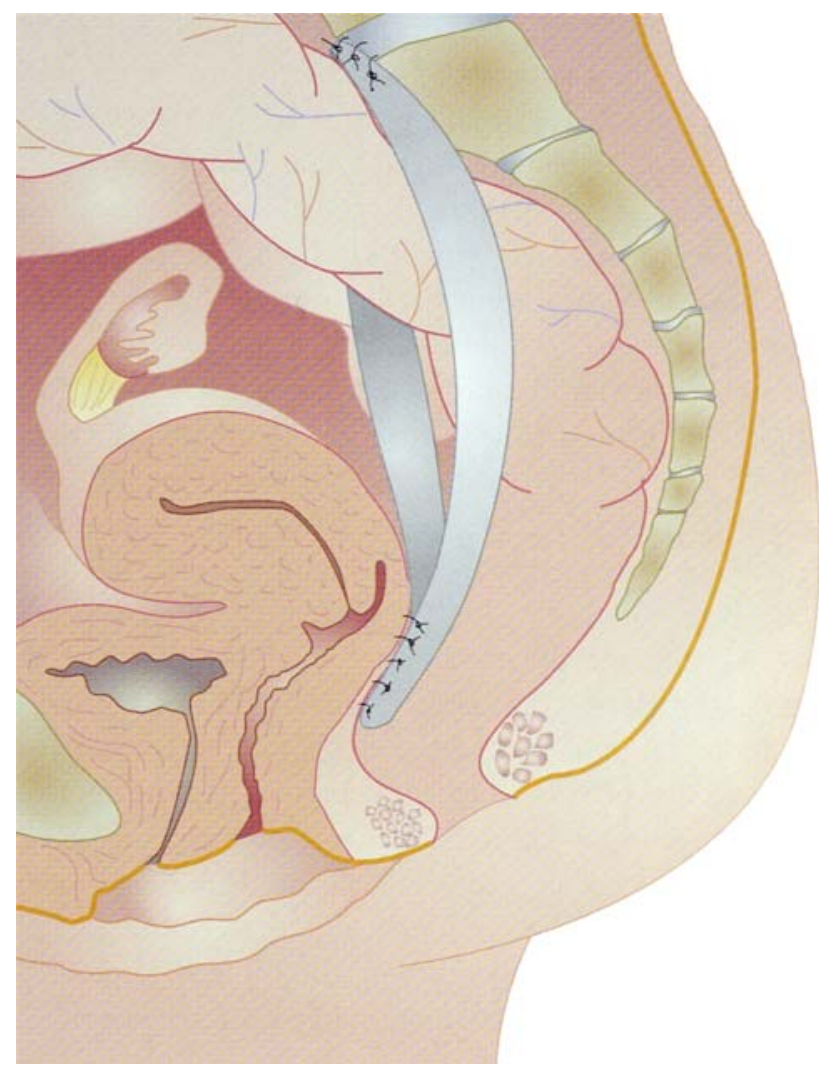

Fig. 3 Technique of rectovaginopexy

position. When the uterus was still present, a transabdominal suture was placed through the corpus of the uterus and knotted extracorporeal resulting in an enhanced view in the pelvic cavity. Adhesiolysis was performed with standard laparoscopic instruments at this time in the procedure if necessary. The surgical cart was then positioned between the patient's legs and the robotic arms connected to the 8-mm robotic ports and camera port, respectively.

Dissection of the peritoneum started on the right side of the rectum using a "Cadiere" type grasper through the left robotic port and an electro cautery hook through the right port. The peritoneum on both sides of the rectum and in the recto/uterine pouch was incised. The peritoneum was dissected from the underlying structures in the lateral and caudal directions. Thereby, no nerves were endangered. The space between the rectum and vagina was then dissected in the avascular plane, down to the level of the pelvic floor. After doing so, the rectum is mobile enough and the part of the rectum originally situated at the bottom of the Douglas pouch can be brought up to the promontory. Next, a passage was created behind the perirectal fat in the avascular plane, and a $20 \times 1 \mathrm{~cm}$ PTFE (Gore-tex) strip was pulled behind the rectum from left to right The promontory was denuded from overlying peritoneum, fat, and connective tissue over a small area. The strip was fixated to the promontory with non-resorbable sutures. Both arms of the strip were now sutured to the anterolateral side of the stretched rectum, as low as possible which is well below the original bottom of the Douglas pouch. Closure of the space between the rectum and vagina to prevent or treat a uterine or vaginal vault prolapse was achieved by suturing the insertions of the utero-sacral ligaments to the upper edge of the PTFE strip with non-resorbable sutures. The peritoneum was then trimmed and reefed by a continuing running resorbable suture, thereby sealing the area of reconstruction and further preventing an enterocele.

\section{Data collection}

Robotic system set-up time, intra-operative and post-operative complications, blood loss, operating time, and hospital stay were collected for each patient. Patients were followed for a minimum of one year after surgery by regular visits to our out-patients clinic.

\section{Statistics}

All statistical analyses were performed using SPSS version 12.0.1 (SPSS, Chicago, IL, USA). Data are expressed as median (range).

\section{Results}

In the period January 2005 to June 2006, we have gained experience with robot-assisted rectovaginopexy in 15 patients. All these operations were completed laparoscopically with support of the da Vinci robotic system. In one patient, a total robotic system failure was encountered after which the procedure had to be performed with standard laparoscopic equipment. Patient characteristics are depicted in Table 1. Preoperative work-up included defaecogram and proctoscopy in all patients, combined anorectal endoscopy and manometry in four, and colonoscopy in one.

Table 1 Patient characteristics at baseline

\begin{tabular}{ll}
\hline Characteristic & Result \\
\hline Duration of symptoms (months) & $33(12-120)$ \\
Male/female $(n)$ & $1 / 14$ \\
Age (years) & $62(33-72)$ \\
Height $(\mathrm{cm})$ & $165(158-195)$ \\
Weight $(\mathrm{kg})$ & $70(54-95)$ \\
BMI & $24.9(20.9-33.9)$ \\
Indication for rectovaginopexy $(n)$ & \\
Rectocele and rectal prolapse & 4 \\
Enterocele and rectal prolapse & 6 \\
Rectocele and enterocele and rectal prolapse & 5 \\
\hline
\end{tabular}

Results are presented as median (range) unless otherwise noted 
Median robot set-up time was 10 min (3-15) and median skin-to-skin operating time was $160 \mathrm{~min}$ (120-180). Median blood loss was $50 \mathrm{cc}(0-300)$. All patients resumed oral feeding within 2-3 days of surgery and median hospital stay was four days (2-9). No in-hospital complications occurred and there was no mortality.

At follow-up two patients needed surgical reintervention $(13 \%)$. One patient was operated for recurrent enterocele and rectocele which manifested one week after surgery. At operation, the fixation of the strip to the sacral promontory appeared to be dehiscent. In another patient an incisional hernia at the camera port occurred three months after surgery. Overall, 13 patients showed favourable results after surgery $(87 \%)$. One patient still experienced faecal incontinence because of an ineffective anal sphincter and one patient complained of severe abdominal pain with no obvious cause. No cases of postoperative newly developed constipation occurred.

\section{Discussion}

The results of this relatively small study of robot-assisted laparoscopic rectovaginopexy for overt and occult rectal prolapse support the safety and feasibility of using a robotic system in this relatively advanced endoscopic procedure. These results support the only report yet published on robot-assisted surgery for rectal prolapse [16].

Rectal prolapse is a distressing condition that is associated with faecal incontinence in $50-70 \%$ of patients [1]. In complete rectal prolapse, the rectal adaptation to distension is obviously impaired, which may contribute to faecal incontinence. There is a higher incidence of prolapse in women and this is strongly correlated with age. Over $50 \%$ of female patients with rectal prolapse are over the age of 70 years [2]. Complications associated with rectum prolapse, such as strangulation, incarceration, and rupture, have been described but are extremely rare [2]. The aim of treatment is twofold:

1 to control the prolapse, and

2 to restore continence and prevent constipation from impaired evacuation.

Optimally, the goal should be to restore normal anatomic configuration and improve the functional outcome. The operation of choice should be characterised by minimal morbidity and mortality with regard to the condition of the patient. Next, the patient's expectation of the functional results of the surgical treatment should be taken into account.

Many abdominal and perineal techniques have been described, differing mainly in the extent of rectal mobilisation, rectal fixation methods, and the inclusion or exclusion of bowel resection [15, 17-20]. The potential advantage of abdominal procedures is fixation of the rectum in a more appropriate anatomic location without sacrifice of the compliant rectal reservoir. In addition, these approaches are generally considered superior because of the lower recurrence rates and better functional results [21]. Perineal approaches that either resect the rectal ampulla with a coloanal anastomosis or plicate the rectal wall are felt to be indicated in older, high-risk patients because of less burden on the cardio-respiratory system. These procedures, however, are associated with worse functional results and higher recurrence rates [2].

The laparoscopic approach for rectal prolapse will potentially reduce pain, shorten hospital stay, and should lead to earlier recovery and faster return to work. Recently, this again was demonstrated in a study focussing on elderly patients with significant comorbidity undergoing laparoscopic rectal prolapse surgery [22]. However, suture rectopexy is a rather demanding procedure when performed as a laparoscopic procedure, because of the large amount of sutures that need to be placed. The introduction of robotic surgery for the repair of rectal prolapse may contribute to the feasibility of the rectovaginopexy as this procedure demands careful dissection at the sacral promontory and in the rectovaginal plane. Furthermore, extensive suturing in the Douglas pouch is necessary, essentially underlining the additive value of a robotic system.

Although laparoscopic techniques for the treatment of rectal prolapse have been described in several studies, only one report focussing on robot-assisted laparoscopic rectopexy has been published so far [16]. Furthermore, there are no large randomized controlled trials comparing different surgical techniques for rectal prolapse; only one small randomized clinical trial has been published on laparoscopic versus open rectopexy [12]. In that trial on 20 patients with laparoscopic repair of a full-thickness rectal prolapse compared with 20 patients with conventional operation (Ripstein rectopexy), favourable results were demonstrated for the laparoscopic group in terms of subjective and objective outcome. However, long-term results of laparoscopic rectopexy in large prospective series are scarce. D'Hoore et al. reported on 42 patients with a laparoscopic ventral rectopexy for total rectal prolapse with a median follow-up of 61 (range 29-98) months [10]. No major postoperative complications occurred and late recurrences of the prolapse were detected in two patients. Furthermore, in 28 of 31 patients with incontinence in this series a significant improvement in outcome was achieved and symptoms of obstructed defecation were resolved in 16 of 19 patients. They therefore concluded that laparoscopic ventral rectopexy is an effective technique without causing severe postoperative constipation.

As for the implementation of robotics in the laparoscopic approach for rectopexy, no clear results have yet been 
published. The role of robotic systems in these surgical procedures remains therefore to be studied. Numerous centres around the world are gaining experience with the robotassisted approach at this point in time but objective results on the advantage of these systems in the surgical repair of rectal prolapse are awaited.

In conclusion, this cohort study on robot-assisted laparoscopic rectovaginopexy has demonstrated that the use of a robotic system during rectal prolapse surgery is safe and, furthermore, may support the surgeon during dissection in the rectovaginal plane and during suturing at the level of the sacral promontory and Douglas pouch. The additional value of these systems, in our perspective, has to be preserved for those surgical procedures that require delicate tissue dissection and suturing. However, the lack of highlevel clinical evidence of the benefits of robot assistance stresses the need for objective data demonstrating the improvement in quality of care using these tools. Therefore, objective proof of the benefit of these new potential applications of robot-assisted surgery and the subjectively perceived additive value of robotic assistance will have to be published for robot-assisted laparoscopic rectovaginopexy also.

Open Access This article is distributed under the terms of the Creative Commons Attribution Noncommercial License which permits any noncommercial use, distribution, and reproduction in any medium, provided the original author(s) and source are credited.

\section{References}

1. Madbouly KM, Senagore AJ, Delaney CP et al (2003) Clinically based management of rectal prolapse. Surg Endosc 17:99-103

2. JJB van Lanschot (ed) (2004) Integrated medical and surgical gastroenterology. Bohn Stafleu Van Loghum, Houten

3. Madiba TE, Baig MK, Wexner SD (2005) Surgical management of rectal prolapse. Arch Surg 140:63-73

4. Benoist S, Taffinder N, Gould S et al (2001) Functional results two years after laparoscopic rectopexy. Am J Surg 182:168-173

5. Zittel TT, Manncke K, Haug S et al (2004) Functional results after laparoscopic rectopexy for rectal prolapse. J Gastrointest Surg 4:632-641

6. Kellokumpu IH, Vironen J, Scheinin T (2000) Laparoscopic repair of rectal prolapse: a prospective study evaluating surgical outcome and changes in symptoms and bowel function. Surg Endosc $14: 634-640$

7. Kessler H, Jerby BL, Milsom JW (1999) Successful treatment of rectal prolapse by laparoscopic suture rectopexy. Surg Endosc 13:858-861

8. Boccasanta P, Venturi M, Reitano MC et al (1999) Laparotomic vs. laparoscopic rectopexy in complete rectal prolapse. Dig Surg 16:415-419

9. Boccasanta P, Rosati R, Venturi M et al (1998) Comparison of laparoscopic rectopexy with open technique in the treatment of complete rectal prolapse: clinical and functional results. Surg Laparosc Endosc 8:460-465

10. D'Hoore A, Cadoni R, Penninckx F (2004) Long-term outcome of laparoscopic ventral rectopexy for total rectal prolapse. Br J Surg 91:1500-1505

11. Poen AC, de Brauw M, Felt-Bersma RJ et al (1996) Laparoscopic rectopexy for complete rectal prolapse. Clinical outcome and anorectal function tests. Surg Endosc 10:904-908

12. Solomon MJ, Young CJ, Eyers AA et al (2002) Randomized clinical trial of laparoscopic versus open abdominal rectopexy for rectal prolapse. Br J Surg 89:35-39

13. Kariv Y, Delaney CP, Casilla S et al (2006) Long-term outcome after laparoscopic and open surgery for rectal prolapse: a casecontrol study. Surg Endosc 20:35-42

14. Ruurda JP, Visser PL, Broeders IA (2003) Analysis of procedure time in robot-assisted surgery: comparative study in laparoscopic cholecystectomy. Comput Aided Surg 8:24-29

15. Silvis R, Gooszen HG, van Essen A et al (1999) Abdominal rectovaginopexy: modified technique to treat constipation. Dis Colon Rectum 42:82-88

16. Munz Y, Moorthy K, Kudchadkar R et al (2004) Robotic assisted rectopexy. Am J Surg 187:88-92

17. Brown AJ, Anderson JH, McKee RF et al (2004) Surgery for occult rectal prolapse. Colorectal Dis 6:176-179

18. Brown AJ, Anderson JH, McKee RF et al (2004) Strategy for selection of type of operation for rectal prolapse based on clinical criteria. Dis Colon Rectum 47:103-107

19. Luukkonen P, Mikkonen U, Jarvinen H (1992) Abdominal rectopexy with sigmoidectomy vs. rectopexy alone for rectal prolapse: a prospective, randomized study. Int J Colorectal Dis 7:219-222

20. Keighley MR, Fielding JW, Alexander-Williams J (1983) Results of Marlex mesh abdominal rectopexy for rectal prolapse in 100 consecutive patients. Br J Surg 70:229-232

21. Aitola PT, Hiltunen KM, Matikainen MJ (1999) Functional results of operative treatment of rectal prolapse over an 11-year period: emphasis on transabdominal approach. Dis Colon Rectum 42:655-660

22. Carpelan-Holmstrom M, Kruuna O, Scheinin T (2006) Laparoscopic rectal prolapse surgery combined with short hospital stay is safe in elderly and debilitated patients. Surg Endosc 20:1353-1359 